\title{
Tip of nose tuberculosis: A rare presentation of extra pulmonary tuberculosis
}

\author{
Darshan K. Bajaj ${ }^{1}$, Ajay K. Verma ${ }^{1}$, Riddhi Jaiswal ${ }^{2}$, Surya Kant ${ }^{1, *}$, Anand Patel ${ }^{1}$, Mona Asnani ${ }^{3}$ \\ ${ }^{1}$ Department of Respiratory Medicine, King George's Medical University UP, Lucknow, India; \\ ${ }^{2}$ Department of Pathology, King George's Medical University UP, Lucknow, India; \\ ${ }^{3}$ Department of Obstetrics \& Gynaecology, Era's Lucknow Medical College, Lucknow, India.
}

\begin{abstract}
Summary Tuberculosis is notorious that it affects various sites of the human body and presents in different ways. One of the uncommon or rather rare presentation of extra pulmonary tuberculosis is nasal tuberculosis. The nose apart from its physiological functions also contributes to facial aesthetics and gives a defined appearance and its deformity imparts cosmetic disfigurement and unsightly appearance. Both primary and secondary forms of nasal tuberculosis are rare but should be considered in the differential diagnosis of ulcerative or crusting lesions of the nose. Here we report such a case of nasal tuberculosis, which presented as an ulcerative and crusting lesion over the tip of the nose in a female child. The patient was given antituberculous chemotherapy after establishing the diagnosis and responded well to treatment.
\end{abstract}

Keywords: Nasal, tuberculosis, granuloma

\section{Introduction}

Though tuberculosis has this unwanted honor of being a major global health problem, the involvement of nose, nasopharynx and para nasal sinus by tuberculosis is rare (1). Trauma and atrophic changes may facilitate introducing bacilli into the nasal lining in the otherwise resistant nasal mucosa (2). Giovanni Morgagni, Professor of Anatomy of Italian origin described nasal tuberculosis in 1761 for the first time while reporting an autopsy of a patient with ulceration of nose, palate and nasopharynx who had concomitant pulmonary tuberculosis (3). Herzog described 20 cases of primary nasal tuberculosis among 80 cases of nasal tuberculosis in the $18^{\text {th }}$ century (3) and Butt described 35 cases of nasal tuberculosis in a review of the $20^{\text {th }}$ century medical literature (4). In this paper, we report a case of nasal tuberculosis manifesting as an ulcerative lesion on tip of nose and we review the relevant literature.

Released online in J-STAGE as advance publication March 10, 2016.

*Address correspondence to:

Dr. Surya Kant, Department of Respiratory Medicine, King George's Medical University UP, Lucknow, India.

E-mail: editor_skant@rediffmail.com

\section{Case report}

A 10 year old female child presented to our department with complaints of a non healing ulcerative and crusted lesion over tip of the nose for almost three months (Figure 1). The patient also had complaints of pus discharge from the lesion and nasal stuffiness. There were no significant constitutional symptoms. She was given a Mantoux test, other routine investigations (Table 1) and was sent to department of otorhinolaryngology for examination of nasal cavity and for obtaining a histopathological specimen from the lesion. Her X-Ray paranasal sinus didn't reveal any significant abnormality. Chest $\mathrm{X}$ ray was grossly normal and sputum for acid fast bacilli was negative on two occasions. Meanwhile she was kept on antibiotics and was asked to report back with histopathology report.

Patient's histopathology from the nasal cavity tissue was suggestive of tuberculosis, with microscopy showing well formed granulomas comprised of epitheloid cells with areas of fibroblastic proliferation entrapping fat as well as fair number of langhans giant cells and evidence of necrosis was also observed (Figure 2 ). Tissue sections were negative for $10 \%$ Potassium Hydroxide $(\mathrm{KOH})$ mount for fungus and fungal culture. The patient's Mantoux test had a induration of 15 $\mathrm{mm}$. She was given anti tuberculous chemotherapy 


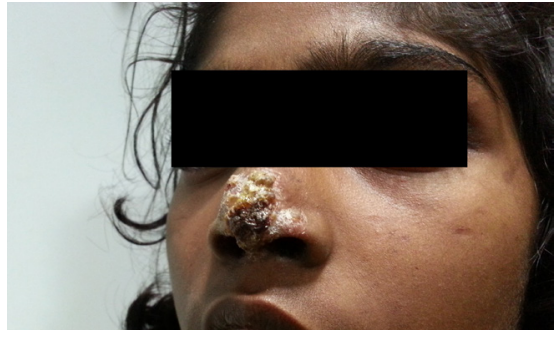

Figure 1. Picture of patient before treatment showing ulcerative $\&$ crusted lesion over Tip of nose.

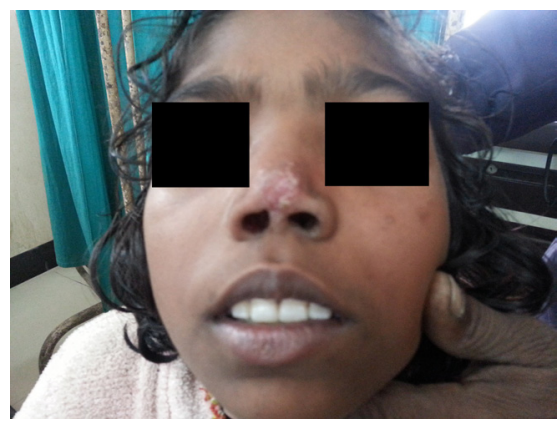

Figure 3. Picture of patient after treatment showing resolution of ulceration \& crusting.

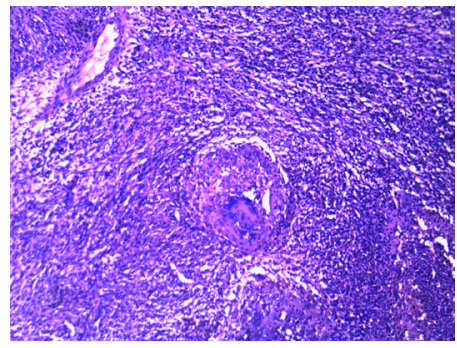

Figure 2. Hematoxylin \& Eosin stained slide showing a well formed epitheloid granuloma with necrosis surrounded by inflammatory cells.

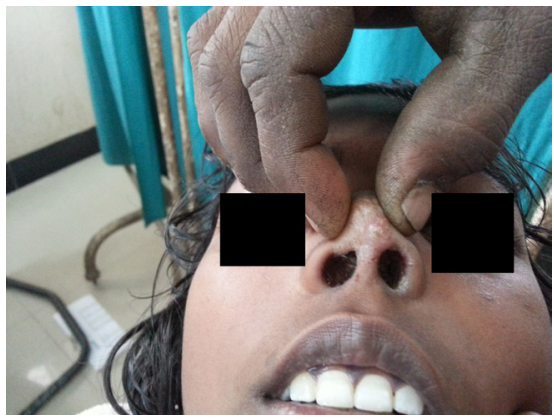

Figure 4. Another picture of patient after treatment showing roomy nasal cavity.

Table 1. Pre treatment evaluation of the patient

\begin{tabular}{ll}
\hline Items & Results \\
\hline Hemogram and Blood sugar & Hb-11.6 g/dl, TLC-9,000/cu/mm, DLC:N-63, L-33,M-02, E-02, RandomBloodSugar-84 mg\% \\
Kidney Function Tests (KFT) & $\begin{array}{l}\text { Blood Urea-23.5 mg/dl } \\
\text { Serum Creatinine-0.8 mg/dl }\end{array}$ \\
Liver Function Tests (LFT) & $\begin{array}{l}\text { Serum Bilirubin (Total-0.5 mg/dl, direct-0.3 mg/dl, indirect-0.2 mg/dl) } \\
\text { SGOT-46 IU/L, SGPT-76 IU/L, SALP-312 IU/L }\end{array}$ \\
Chest X ray & Normal \\
X ray Para-nasal sinus & No significant Abnormality \\
Sputum For Acid Fast Bacilli & Negative on two occasions \\
\hline
\end{tabular}

comprising of Rifampicin, Isoniazid, Ethambutol and Pyrazinamide according to her body weight and was directed for follow up at two months. Patient responded to the treatment and the lesion considerably improved with resolution of the nasal blockade and pus discharge (Figure 3 and Figure 4).

\section{Discussion}

Extra pulmonary tuberculosis has been on the rise during the past few decades constituting around 15 to 20 percent of tuberculosis cases in immunocompetent subjects and more than 50 percent of cases in human immunodeficiency virus (HIV) positive and other immunocompromised states. The most common sites affected by it are lymph nodes followed by pleura although other sites including bones, genito urinary system, abdomen, and meninges are also involved in a fair number of cases.

Extra pulmonary tuberculosis manifesting on unusual sites is a diagnostic challenge for the treating physician and nasal tuberculosis is one of them because the signs and symptoms are nonspecific (5). Nasal tuberculosis is usually secondary either to pulmonary tuberculosis or to lupus vulgaris of facial skin (6). Primary disease is rare. Ciliary movement, the bactericidal effect of nasal secretions, filtering by nasal vibrissae and the inherent resistance offered by nasal mucosa can explain the lower occurrence of nasal tuberculosis (5). Differential diagnosis can include various infectious as well as non-infectious causes which are shown (2) (Table 2).

Most cases of nasal tuberculosis are unilateral and lesions can be proliferative, infiltrative or ulcerative 
Table 2. Differential diagnosis of Granulomatous lesions of nose

\begin{tabular}{ll}
\hline Items & Diagnosis \\
\hline Infectious & $\begin{array}{l}\bullet \text { Bacterial: Rhinoscleroma, Leprosy, Syphilis, Yaws } \\
\bullet\end{array}$ \\
& $\bullet$ Pungal: Mucormycosis, Aspergillois, Blastomycosis, Histoplasmosis \\
Non infectious & $\bullet$ Wegener's Granulomatosis, Sarcoidosis \\
Malignant & $\bullet$ Midline Granuloma \\
Miscellaneous & $\bullet$ Trauma, Cocaine abuse \\
\hline
\end{tabular}

(4). Pulmonary and extra pulmonary tuberculosis at adjacent sites have been reported in cases of nasal tuberculosis though rarely constituting roughly $2-6 \%$ of extrapulmonary cases $(7,8)$.

Sometimes the role of computed tomography (CT) and magnetic resonance imaging (MRI) come into play in evaluating the various kinds of nasal cavity masses as they may differentiate the contents, and they may localize the lesion by different contrast enhancement patterns. In fully ossified lesions like in rhinolitis, osteomas and fibrous dysplasias diffuse hypointensity on MRI and sclerosis on CT hints towards benign pathology. Cysts, secretions and mucoceles appear as smoothly bordered non enhanced lesions. Masses with different enhancements with smooth borders hint towards nerve tissue or minor salivary gland tumors. Uniform contrast enhancement can be observed in hemangioma or angiofibroma. Destruction, invasion and necrosis may be observed with malignant neoplasms, granulomatous reactions, metastasis and fungal infections (9). A definitive diagnosis is established by isolating tuberculous bacilli from tissue specimens obtained for biopsy or from surgery. Management of this condition in the past was more or less symptomatic before the introduction of anti-tubercular chemotherapy including irrigations with alkaline antiseptics, use of hydrogen peroxide, lactic acid, trichloroacetic acid, use of galvanocautery, use of astringents and pyrogallic ointments etc. The treatment of nasal tuberculosis is the same as for the other extrapulmonary sites, it can be accompanied by surgical intervention with cosmetic correction depending on the case and extent of involvement.

The following three types of sinonasal tuberculosis have been described in the literature $i$ ) mucosal involvement leading to formation of polyps with minimal pus discharge; ii) bony involvement and fistula formation with abundant discharge of acid-fast bacilli; and iii) hyperplastic changes with formation of tuberculoma. In the case of sinus involvement the most commonly affected is the maxillary sinus (10).

Few important differential diagnoses can be considered while dealing with a case of nasal granuloma like in Rhinosporidiasis histological examination of the lesion shows characteristic appearance of sporangia, oval or round in shape filled with spores which may be seen bursting through its chitin walls (11). Rhinoscleroma is another differential diagnosis with demonstration of characteristic Mikulicz cells in its histology (12). In cases of aspergillosis of nose the histological examination of tissue demonstrates infiltrating aspergillus hyphae (13). Histology of sarcoidosis demonstrates non caseating granulomas. Midline granuloma is a lethal malignant condition characterized by angiocentric and angiodestructive growth patterns with extensive mucosal ulceration and lymphomatous infiltrate $(14,15)$.

Many times the diagnosis is delayed and it may lead to further involvement of the adjacent structures and cosmetic disfigurement but fortunately in our patient there was a superficial ulcerative and crusting lesion over the tip of her nose which probably prompted her parents to seek early consultation and so based on clinical suspicion followed by histopathological confirmation with demonstration of epitheloid granulomas, necrosis and other supportive features suggestive of tuberculosis the patient was started on antitubercular chemotherapy. The patient responded well to the treatment with resolution of her lesion and improvement in general well being and didn't require any sort of cosmetic surgery.

\section{Conclusion}

Extra pulmonary tuberculosis has a wide spectrum of presentation and may manifest with constitutional symptoms, which sometimes are the only diagnostic clue. Sometimes it is not possible to do a bacteriological and histopathological confirmation of the diagnosis and treatment is given based on clinical suspicion. Just like pulmonary tuberculosis, the patients of extra pulmonary tuberculosis respond well to antitubercular chemotherapy in most cases, however some cases may proceed to complications or may be left with remnants of the primary disease.

Our patient was a case of tuberculosis of tip of the nose, which is a rare presentation of extra pulmonary tuberculosis and was successfully treated with standard antitubercular chemotherapy. Early diagnosis and treatment are the cornerstone of management and can prevent complications. 


\section{Acknowledgements}

The authors wish to acknowledge the patient and attendants for their support.

\section{References}

1. Menon K, Bem C, Gouldesbrough D, Strachan DR. A clinical review of 128 cases of head and neck tuberculosis presenting over a 10-year period in Bradford, UK. J Laryngol Otol. 2007; 121:362-368.

2. Hyams VJ. GM English, Otolaryngology. Philadelphia: JB Lippincott; Pathology of the nose and paranasal sinuses. 1991; pp. 24-25.

3. Waldman SR, Levine HL, Sebek BA, Parker W, Tucker HM. Nasal tuberculosis: A forgotten entity. Laryngoscope. 1981; 91:11-16.

4. Butt AA. Nasal tuberculosis in the 20th century. Am J Med Sci. 1997; 313:332-335.

5. Hughes RG, Drake-Lee A. Nasal manifestations of granulomatous disease. Hosp Med. 2001; 2:417-421.

6. Howard D. Nonhealing granulomas. In: Mackay IS, Bull TR, editors. Scott Brown's Otolaryngology: Rhinology. 6th ed. Oxford: Butterworth and Heinemann; 1997. p. 4/20:1-11.

7. Dixit R, Sharma S, Nuwal P. Tuberculosis of oral cavity. Indian J Tuberc. 2008; 55:51-53.

8. Ghatak S, Jana T, Bandyopadhyaya S, Biswas SK. Primary oropharyngeal tuberculosis causing destruction of uvula- A rare presentation. Int J Paed Otorhinolaryngol. 2010; 5:91-92.

9. Yildirim D, Saglam O, Gurpinar B, Ilica T. Nasal cavity masses: Clinico-radiologic collaborations, differentiated diagnosis by special clues. Open Journal of Medical Imaging. 2012; 2:10-18.

10. Kakeri AR, Patel AH, Walikar BN, Watwe MV, Rashinkar SM. A case of tuberculosis of maxillary sinus. Al Ameen J Med Sci. 2008; 1:139-41.

11. Madke B, Mahajan S, Kharkar V, Chikhalkar S, Khopkar U. Disseminated cutaneous with nasopharyngeal rhinosporidiosis: Light microscopy changes following dapsone therapy. Australas J Dermatol. 2011; 52:e4-e6.

12. Yigla M, Ben Izlak O, Oren I, Hashman N, Lejbkowicz F. Laryngo-tracheobronchial involvement in a patient with nonendemic rhinoscleroma. Chest. 2000; 117:1795-1798.

13. Katzenstein AL, Sale SR, Greenberger PA. Pathologic findings in allergic aspergillus sinusitis. Am J Surg Pathol. 1983; 7:439-443.

14. Metgud RS, Doshi JJ, Gaurkhede S, Dongre R, Karle R. Extranodal NK/T-cell lymphoma, nasal type (angiocentric T-cell lymphoma): A review about the terminology. J Oral Maxillofac Pathol. 2011; 15:96-100.

15. Mehta V, Balachandran C, Bhat S, Geetha V, Fernandes D. Nasal NK/T cell lymphoma presenting as a lethal midline granuloma. Indian J Dermatol Venereol Leprol. 2008; 74:145-147.

(Received November 26, 2015; Revised January 29, 2016; Accepted January 31, 2016) 